\title{
Introducing the 'Psychosomatic Screening Questionnaire - 29' (PSSQ-29): Reliability and Validity in an Epidemiological Sample of 1,158 Participants in Greece during the COVID-19 Domestic Lockdown
}

\author{
Georgios Pilafas $^{1}$, Alexandra Prouzou ${ }^{1}$, Nefeli Paraskevi Strongylaki ${ }^{2}$, \\ Despina Menti ${ }^{3}$, Georgios Lyrakos ${ }^{4}$ \\ ${ }^{1} \mathrm{MSc}, \mathrm{MSc}$, Research Associate, CityU Research Center, Psychology Laboratory, City Unity College, Athens, \\ Greece \\ ${ }^{2} \mathrm{MSc}, \mathrm{MBPsS}$, Research Associate, CityU Research Center, Psychology Laboratory, City Unity College, \\ Athens, Greece \\ ${ }^{3}$ PgDip, MSc, PhD, CPsychol, EuroPsy, Psychology Lecturer, Department of Psychology, Cardiff Metropolitan \\ University at City Unity College, Athens, Greece \\ ${ }^{4} \mathrm{MSc}, \mathrm{MPH}, \mathrm{PhD}$, PostDoc, 'MSc Health Psychology' Program Director, Department of Psychology, Cardiff \\ Metropolitan University at City Unity College, Athens, Greece; and 'Clinical Health Psychologist', Psychiatric \\ Department, General Hospital of Nikaia 'Ayios Panteleimon', Athens, Greece
}

Corresponding Author: Georgios Pilafas

\begin{abstract}
Background: Although the field of psychosomatics is one of the oldest in behavioral health science, there may be a total absence of a self-reported psychometric scale that measures simultaneously mental and somatic symptoms. Thus, the present study introduces a new 29-item psychometric tool, named 'Psychosomatic Screening Questionnaire - 29' (PSSQ-29).

Aim: PSSQ-29 was designed in order to provide a reliable and valid scale that measures both mental and somatic symptoms in a single questionnaire.

Methods \& Materials: Research methods were employed and statistical analysis was performed to test 'face validity', 'content validity', 'internal validity', 'construct validity' and 'predictive validity' of PSSQ-29, while a 'factor analysis' also took place. The rest materials that were used in this study include the Greek versions of the 'Nicholson McBride Resilience Questionnaire' and the 'Acute Stress Disorder Scale'.

Results: The highlights of the analysis include a $95.5 \%$ reliability ratio, a strong correlation with 'psychological resilience' and 'acute stress disorder', while 59\% of variance of PSSQ-29 was predicted by the levels of acute stress of the same sample at the same time.

Discussion: The findings of this study support the use of PSSQ-29 by health professionals and researchers in the foreseeable future. What is more, PSSQ-29 may provide much assistance in research regarding multi-morbid conditions in Behavioral Medicine.

Conclusion: Finally, PSSQ-29 was found both reliable and valid in the Greek population. Amongst many proposed ways of use, it is highly recommended to be used at any new 'mass panic' situation in Greece.
\end{abstract}

Key words: Psychosomatic Screening Questionnaire; PSSQ-29; Psychometrics; COVID-19; Greece 


\section{INTRODUCTION}

The present work is part of a larger study that was undertaken during the 'Corona-Virus Disease 2019' (COVID-19) outbreak in Greece. A part of the larger study included the intention to measure the statistical levels of both mental and somatic symptoms, as these interact in the rationale of 'Psychosomatics'. The background review of the research team aimed to find a valid and reliable self-reported tool that measures psychosomatic symptoms. The result of the review led to the conclusion that existing questionnaires include items that either measure mental or somatic symptoms. This practically excludes any of these tools from psychosomatic research, since the theory of psychosomatics considers mental and somatic symptoms as one. ${ }^{[1]}$ Altogether, led the research team to create a new self-reported psychometric tool that measures both mental and somatic symptoms, and which we introduce in this article.

The name of this new self-reported questionnaire is 'Psychosomatic Screening Questionnaire-29' (PSSQ-29), and it consists of 29 items. PSSQ-29 was designed based on the rationale of 'psychosomatic disorders', as these are discussed in Behavioral Medicine ${ }^{[1-4]}$ as well as on the 'biopsychosocial approach' of illnesses ${ }^{[5,6]}$ and the ideology of 'neutral monism' ${ }^{[7-9]}$

\subsection{Background}

The cornerstone of PSSQ-29 is 'stress', and more specifically the sympathetic nervous adaptation. Briefly, based on Hans Selye's [10] initial works, 'stress' is defined as the psychoneuroendocrinological response against any stressors. [11] After the presentation of any stressor, such as a 'life-threatening event' or a 'natural disaster', the human body responses with the activation of the 'HypothalamicPituitary-Adrenal (HPA) Axis' of the 'Sympathetic-Nervous-System' (SNS). [12] This response is known to keep human organs in the well-renowned 'fight-or-flight' response. ${ }^{[12,13]}$ Any activation of the HPA
Axis results in the production of hormones that regulate the body. ${ }^{[14]}$ From research in previous decades, it is evident that stress and emotions interact. ${ }^{[15-18]}$ A large portion of these interactions may be found in 'psychosomatic disorders'. Historically, psychosomatic disorders have been referenced as conditions which reflect both mental and somatic disorders, while they are triggered by stressful life phenomena. [1] Furthermore, in studies related to psychosomatic medicine, an acute and prolonged exposure to stress may lead to several somatic disorders. Such disorders include for instance 'peptic ulcers', [19] 'skin acne' [20] and 'Cardiovascular-Disease' (CVD). ${ }^{[21]}$ The stress response is also of great concern in mental health, since it interacts with emotions, memory and cognition in what are commonly referred as 'adaptive' and 'maladaptive' cognitive appraisals. ${ }^{[22]}$

To proceed, in Greece the domestic government established public measures against the spread of COVID-19 from February 24th, 2020. [23] Government's measures were strictly enforced by the police. Between March 23rd and May 4th of 2020 public and private businesses were closed and the Greek economy was 'locked down'. Thus, much uncertainty for the future was raised. The financial situation was discussed as critical in the mass media of communication. Professional opinions of epidemiologist and medical doctors regarding the risks of COVID-19 were often referenced on the news, newspapers, and the web. Moreover, from March $23^{\text {rd }}$ to May $4^{\text {th }}$ of 2020 citizens were enforced to remain in their houses, since in person social interactions and transportations were not allowed for any reason. The only exceptions included some very specific circumstances such as visiting a medical doctor or the local food store. Any physical exercise was limited, and citizens were allowed to exercise outdoors in a close proximity to their house for less than one hour. 
Georgios Pilafas et.al. Introducing the 'psychosomatic screening questionnaire - 29' (PSSQ-29): reliability and validity in an epidemiological sample of 1,158 participants in Greece during the COVID-19 domestic lockdown.

\subsection{Hypothesis \& Aim}

Our research team hypothesized that the COVID-19 outbreak in the country and the measures that were in effect from March 23rd and May 4th of 2020, were a common stressor for the Greek population. In authors' standpoint, this stressor was likely to increase sympathetic nervous adaptation for a prolonged time, and thus to increase symptoms of psychosomatic disorders. As already reported, in order for the authors to measure any psychosomatic symptom for the larger study, PSSQ-29 was designed. PSSQ was expected to show high validity and reliability, while it was also expected to present a negative correlation with a selfreported scale that measures 'psychological resilience' and a positive correlation with again, a self-reported scale that measures 'acute stress disorder'.

Accordingly, the aim of developing PSSQ-29 was firstly to use the new scale in our larger study, and secondly to create and deliver a valid and reliable self-reported psychometric tool that can accurately measure the levels of both mental and somatic symptoms in a single scale. In this article, it is provided (i) the design process of PSSQ-29, (ii) the analysis and (iii) the discussion regarding the results that were observed upon the validity and reliability of PSSQ-29 in the larger epidemiological study in Greece.

\section{METHODS}

\subsection{Participants, Conditions \& Statistical Power}

\begin{tabular}{|c|c|c|c|c|c|}
\hline \multicolumn{6}{|c|}{ Table 1. Demographic details of the participants of the study. } \\
\hline Main Variable & Variable's Subcategories & $\begin{array}{l}\text { Total }(\%) \\
N=1,158^{a}\end{array}$ & $\begin{array}{c}\text { Males }(\%) \\
\mathrm{n}=\mathbf{2 8 0},(\mathbf{2 4 . 2 \%})\end{array}$ & $\begin{array}{c}\text { Females }(\%) \\
n=876,(75.6 \%)\end{array}$ & Missing \\
\hline \multirow[t]{4}{*}{ Education } & & & & & - \\
\hline & School Level, $(\%)$ & $399,(34.5 \%)$ & $110,(39.3 \%)$ & $289,(33 \%)$ & \\
\hline & Undergraduate Degree, $(\%)$ & $402,(34.7 \%)$ & $85,(30.35 \%)$ & $316,(36.1 \%)$ & \\
\hline & Postgraduate Degree, $(\%)$ & $357,(30.9 \%)$ & $85,(30.35 \%)$ & $271,(30.9 \%)$ & \\
\hline \multirow[t]{8}{*}{ Marital Status } & & & & & - \\
\hline & Single, $(\%)$ & $299,(25.8 \%)$ & $111,(39.6 \%)$ & $188,(21.5 \%)$ & \\
\hline & In relationship, $<5$ years, $(\%)$ & $123,(10.6 \%)$ & $21,(7.5 \%)$ & $101,(11.5 \%)$ & \\
\hline & In relationship, $>5$ years, $(\%)$ & $74,(6.4 \%)$ & $17,(6.1 \%)$ & $56,(6.4 \%)$ & \\
\hline & Married, $(\%)$ & $508,(43.9 \%)$ & $101,(36.1 \%)$ & $407,(46.5 \%)$ & \\
\hline & Separated, $(\%)$ & $25,(2.2 \%)$ & $2,(0.7 \%)$ & $23,(2.6 \%)$ & \\
\hline & Divorced, $(\%)$ & $113,(9.8 \%)$ & $27,(9.6 \%)$ & $86,(9.8 \%)$ & \\
\hline & Widowed, (\%) & $16,(1.4 \%)$ & $1,(0.4 \%)$ & $15,(1.7 \%)$ & \\
\hline \multirow[t]{6}{*}{ Children } & & & & & - \\
\hline & None, $(\%)$ & $527,(45.5 \%)$ & $157,(56.1 \%)$ & $368,(42 \%)$ & \\
\hline & $1,(\%)$ & $201,(17.4 \%)$ & $44,(15.7 \%)$ & $157,(17.9 \%)$ & \\
\hline & $2,(\%)$ & $348,(30.1 \%)$ & $67,(23.9 \%)$ & $281,(32.1 \%)$ & \\
\hline & $3,(\%)$ & $69,(6 \%)$ & $9,(3.2 \%)$ & $60,(6.8 \%)$ & \\
\hline & $\geq 4,(\%)$ & $13,(1.1 \%)$ & $3,(1.1 \%)$ & $10,(1.1 \%)$ & \\
\hline \multirow[t]{11}{*}{ Occupation } & & & & & - \\
\hline & Unemployed, (\%) & $84(7.3 \%)$ & $13,(4.6 \%)$ & $71,(8.1 \%)$ & \\
\hline & School \& University Student, (\%) & $163(14.1 \%)$ & $48,(17.6 \%)$ & $114,(13 \%)$ & \\
\hline & Self-Employed/Freelancer, $(\%)$ & $142(12.3 \%)$ & $35(12.5 \%)$ & $107,(12.2 \%)$ & \\
\hline & Public Servant, $(\%)$ & $214(18.5 \%)$ & $57,(20.4 \%)$ & $157,(17.9 \%)$ & \\
\hline & Employee at the private sector, $(\%)$ & $315(27.2 \%)$ & $73,(26.1 \%)$ & $242,(27.6 \%)$ & \\
\hline & Health Professional, $(\%)$ & $133(11.5 \%)$ & $29,(10.4 \%)$ & $103,(11.8 \%)$ & \\
\hline & Security \& Armed Forces, (\%) & $9(0.8 \%)$ & $5,(1.8 \%)$ & $4,(0.5 \%)$ & \\
\hline & Rentier/Landlord, (\%) & $13(1.1 \%)$ & $1,(0.4 \%)$ & $12,(1.4 \%)$ & \\
\hline & Retired, $(\%)$ & $76(6.6 \%)$ & $16,(5.7 \%)$ & $60,(6.8 \%)$ & \\
\hline & Disability Pension, (\%) & $9(0.8 \%)$ & $3,(1.1 \%)$ & $6,(0.7 \%)$ & \\
\hline \multirow[t]{5}{*}{ Income $^{\mathrm{b}}$} & & & & & $1^{\mathrm{c}}$ \\
\hline & $\leq 10,000 €,(\%)$ & $379,(32.8 \%)$ & $67,(24 \%)$ & $310,(35.4 \%)$ & \\
\hline & $10,001-20,000 €,(\%)$ & $401,(34.7 \%)$ & $118,(42.1 \%)$ & $283,(32.3 \%)$ & \\
\hline & $20,001-30,000 €,(\%)$ & $174,(15 \%)$ & $44,(15.7 \%)$ & $130,(14.8 \%)$ & \\
\hline & $\geq 30,001 €,(\%)$ & $203,(17.5 \%)$ & $50,(17.9 \%)$ & $153,(17.5 \%)$ & - \\
\hline \multirow[t]{6}{*}{ Residence } & & & & & - \\
\hline & Athens, $(\%)$ & $934,(80.7 \%)$ & $237,(84.6 \%)$ & $695,(79.3 \%)$ & \\
\hline & Thessaloniki, (\%) & $24,(2.1 \%)$ & $3,(1.1 \%)$ & $21,(2.4 \%)$ & \\
\hline & Rest Mainland Greece, $(\%)$ & $118,(10.2 \%)$ & $27,(9.6 \%)$ & $91,(10.4 \%)$ & \\
\hline & Greek Islands, $(\%)$ & $66,(5.7 \%)$ & $11,(3.9 \%)$ & $55,(6.3 \%)$ & \\
\hline & Other, non specified, $(\%)$ & $16,(1.4 \%)$ & $2,(0.8 \%)$ & $14,(1.6 \%)$ & \\
\hline \multicolumn{6}{|c|}{$\begin{array}{l}\text { Notes: } \\
\text { a Out of the } 1,158 \text { participants } 2 \text { of them }(0.2 \%) \text { did not declare their gender } \\
{ }^{b} \text { This variable shows the amount of the total annual income in the household after the contribution of all members } \\
{ }^{c} \text { The single missing case was located in the men's group }\end{array}$} \\
\hline
\end{tabular}


The aim of the researchers was to recruit the highest possible amount of intellectually healthy adults who could read and answer questions in Greek. There were no restrictions regarding any mental and physical illnesses. The study was promoted on social media and researchers used the 'snowball' sampling method.24 PSSQ-29 was only answered on 'Google Forms'. All participants included in this study (i) had to be electronic literate, (ii) had to have access to a computer, tablet or smartphone and (iii) use social media networks where the study was posted. All participants included in the final sample did meet these criteria. For that reason, in the present study a convenient sample was used. ${ }^{[25]}$

As already discussed in the introduction, the study took place during the COVID-19 outbreak in Greece and the enforcement of obligatory precaution measures against the spread of the disease by the Greek government. All participants provided their answers only between March $23^{\text {rd }}$ and May $4^{\text {th }}$ of 2020.

Eventually, 1,158 Greek adults participated in this study. The age of the participants ranges between 18 and 78 years old, with a mean score of 40.51 ( $\mathrm{SD}=$ \pm 12.8 ). The average days that the participants had been under government's measures is 33.3 (SD $= \pm 7.23$ ). More information upon the demographics of this study is given in Table 1 .

In regard to the sample size of this study, Comrey and Lee ${ }^{[26]}$ discuss that a total number of more than 1,000 participants is 'excellent'. Further, a ratio of 2 up to 20 individuals per item is suggested for such studies, with a minimum of 100 to 250 participants in total. ${ }^{[27]}$ In this study the number of 1,158 participants provides a ratio of 37.35 participants to each respective item of the new questionnaire. What is more, the power of the study was calculated by the use of ' $G$ *Power 3.1 ' software. ${ }^{[28]}$ The amount of participants $(\mathrm{N}=1,158)$ provided to the study an 'a priori' odds ratio of 1.274 and an actual power of $95 \%$.

\subsection{Item Design}

In regard with the design of the items of PSSQ-29, it was reflected that a reliable and valid tool that can measure psychosomatic symptoms has to include items that measure both mental and somatic conditions in the same scale.

For the initial design of the items of PSSQ-29, a member of our research team, who has an academic and professional background in Health Psychology, used the 10th edition of the 'International Classification of Diseases' (ICD-10) ${ }^{[29]}$ and the 'Encyclopedia of Behavioral Medicine'. [11] Conditions that are related to 'mood', 'cognition', 'emotions', 'memory', 'eating', 'somatic' and 'stress' disorders were located into the latter two sources.

In order for the located disorders and their conditions to be used in the design of the items of PSSQ-29 a few criteria were used. The researcher considered as inclusion criteria a clear (i) 'pathophysiology', (ii) 'etiopathology', (iii) 'phenomenology' and (iv) 'objectiveness'. After the inclusion/exclusion process, the symptoms of the conditions were considered one by one. Most symptoms were almost identical, since most conditions overlap. Therefore, any symptom was included only once into the new questionnaire. This process resulted in the creation of an initial 31-item new questionnaire for further statistical evaluation.

All questions started with the statement 'During the social measures against coronavirus, [...]' and continued with the description of the selected symptoms, such as '[...], I noticed that I experience more nightmares'. Each question was measured in a self-reported fashion, 30 by the use of a progressive '0-to-10 scale'. Participants were informed in the instruction section of the new questionnaire and in each question that the scoring pattern is ' $0=$ no symptoms' and ' $10=$ a lot of symptoms, I need professional help the soonest possible'. 


\subsection{Other Measures}

In the present study beyond the new questionnaire, two more questionnaires were used for correlation analysis with PSSQ-29. All questionnaires were answered by the same participants at the same time period. The two questionnaires are as follows.

\subsubsection{Nicholson McBride Resilience Questionnaire}

The first questionnaire concerns the Greek adaptation of Nicholson McBride Resilience Questionnaire (NMRQ). ${ }^{[31]}$ The tool was used in order to measure the level of psychological resilience of the participants and it consists of 12 selfreported items. It is reported as reliable for the Greek population, as Cronbach's alpha was found at .800. ${ }^{[31]}$

\subsubsection{Acute Stress Disorder Scale}

The second self-reported questionnaire that was used is the Greek adaptation of Acute Stress Disorder Scale (ASDS), which illustrates the levels of 'Acute Stress Disorder' (ASD). ${ }^{[32]}$ This psychometric tool includes 19 self-reported items. The Greek adaptation of ASDS presents a high percentage of reliability, as Cronbach's alpha was found at $.925 .^{[32]}$

\subsection{Procedure, Study Design \& Statistical Analysis}

After the ethical approval, the creation of the electronic platform that received the responses on 'Google Forms' and the data collection process, the final answers of the participants were transferred to SPSS version 26 software for further statistical analysis. ${ }^{[33]}$

As it is already known, beyond the aims of the larger study, the researchers decided to test the validity and reliability of the newly designed PSSQ-29. Hence, the design of the present study concerns the later research questions. The testing took place in five respective stages.

Firstly, after the design of the 31 items, the instructions and the scoring patterns of the new questionnaires, 5 individuals with a psychology background, 3 with a non-psychology background and 2 under-aged people who at that juncture were aged 14 years old, were asked to read and confirm to the researchers that they understand the items and what is asked from them to do. The individuals with the psychology background were mainly employed to test the 'content validity' of PSSQ-29 by providing their knowledge upon the design of the items and the conditions that are described in PSSQ-29, while the rest of the individuals the 'face validity' of the new tool. ${ }^{[27]}$

Secondly, after the final data were collected and transferred to SPSS software, the internal validity of PSSQ-29 was tested. More specifically, the correlations between the items of the new questionnaire were tested, as well as the score of Cronbach's alpha was calculated. It was decided by the researchers that no 'test-retest' validity will take place. The later process may present some strong bias in the results, due to 'observational/measurement error', and more specifically due to 'systematic' and 'random' errors. ${ }^{[34]}$ It is worth mentioning that PSSQ-29 contains self-reported items that describe symptoms that are related to sympathetic nervous adaptation and cognition. Hence, the odds for statistical bias in a test-retest validity analysis are likely to be higher than average. ${ }^{[35,36]}$

The next stage of the study included an Exploratory Factor Analysis (EFA). The analysis includes three steps. The first step concerns the 'extraction of items'. All initial 31 items were put for further analysis in order to test how many factors exist in the new questionnaire. Thereinafter, a 'factor loading analysis' was performed through the application of the 'varimax rotation method'. In the last step of EFA, the factors were interpreted by the person who designed the initial items of PSSQ-29. The researcher interpreted the factors according to the description of the symptoms in each item (e.g., nightmares), and how these 
correspond to existing mental and somatic conditions.

Then, construct validity analysis was performed by testing the correlation of PSSQ-29 firstly with ASDS and secondly with NMRQ. It was expected that PSSQ-29 will be positively correlated with ASDS and negatively correlated with NMRQ. This method tested the 'convergent validity' of the new questionnaire. ${ }^{[27]}$

Finally, the 'predictive validity' of PSSQ-29 was tested through a linear regression analysis. ${ }^{[27]}$ To elaborate, the background theory of PSSQ-29 supports that the conditions included in the items are stress-related and that stress is the cornerstone of psychosomatics. In consequence, ASDS was used as the criterion variable and PSSQ-29 as the outcome variable in this linear regression analysis.

\section{RESULTS}

\subsection{Face Validity \& Content Validity}

All of the 8 individuals, including the 5 individuals with a psychology background, the 3 persons with a nonpsychology background and the 2 underaged people aged at 14 years old, did not ask for any further instructions upon the new questionnaire. Therefore, it was considered by the researchers that the instructions, the items and the scoring pattern were fully understood. ${ }^{[37]}$

Psychology experts did not provide any obsession to the epistemonology of the items and PSSQ-29 as a whole. As a result, the content of the items and the overall questionnaire were thought to be consistent to current empirical evidence. ${ }^{[27]}$

\subsection{Internal Validity}

Internal correlation between all initial 31 items of the questionnaire was tested by the application of Pearson's r. ${ }^{[38]}$ The results are given in the Table 2.

'Corrected item-total correlation' analysis on SPSS output showed that 'item 19 ' and 'item 20' scored below the amount of .40 . It was decided by the designer of the
PSSQ-29 to delete these two items from the questionnaire with a view to increase the internal consistency of PSSQ-29. ${ }^{[38]}$

Retrospectively, internal consistency was further tested by the use of Cronbach's alpha coefficient analysis on the remaining 29 items of the questionnaire. The results suggest an overall high reliability of $95.5 \%$ for PSSQ-29. ${ }^{[38]}$

\subsection{Factor Analysis \\ 3.3.1 Extraction of Items}

EFA showed 4 components whose Eigenvalue is at least 1 . This finding suggests that there may be 4 factors in the new questionnaire. ${ }^{[38]}$

\subsubsection{Rotation of Items}

In the rotation process, it was considered in advance that any item had to present at least an absolute value of above .40 in order to be considered in the factor loading analysis. Although EFA had shown 4 components whose Eigenvalue was higher than 1 , the factor loading analysis showed that all items, without any exception, load predominantly factor 1 . The loading scores range from .450 to .780 . It is noteworthy that only 4 items out of the 29 provide some little loading to the rest 3 factors. The range of the loading scores is limited and is found between .401 and .485 , while in all cases these 4 factors provide more loading to factor 1 .

\subsubsection{Interpretation of Factors}

Based on ICD-10, [29] the Encyclopedia of Behavioral Medicine, [11] the stress response of Hans Selye, ${ }^{[10]}$ the works of Richard Lazarus ${ }^{[15]}$ and the results of EFA in the present study, the researcher who designed the initial items of the new questionnaire considered that PSSQ-29 has only one factor that is interpreted as 'psychosomatic symptoms' that occur after the stress response. 


\begin{tabular}{|c|c|c|c|c|c|c|c|c|c|c|c|c|c|c|c|c|c|c|c|c|c|c|c|c|c|c|c|c|c|c|c|}
\hline Q. & 1 & 2 & 3 & 4 & 5 & 6 & 7 & 8 & 9 & 10 & 11 & 12 & 13 & 14 & 15 & 16 & \begin{tabular}{|l}
17 \\
\end{tabular} & 18 & 19 & 20 & 21 & 22 & 23 & 24 & 25 & 26 & 27 & 28 & 29 & 30 & 31 \\
\hline 1 & & 养 & 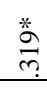 & 养 & 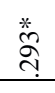 & 尊 & 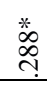 & $\stackrel{*}{\stackrel{*}{m}}$ & 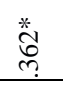 & 蒂 & 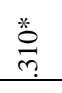 & $\stackrel{*}{\stackrel{*}{m}}$ & 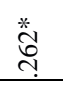 & 䍖 & 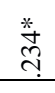 & 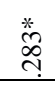 & $\stackrel{*}{\stackrel{2}{m}}$ & 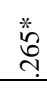 & 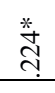 & $\begin{array}{l}\text { * } \\
\stackrel{\infty}{\infty} \\
=\end{array}$ & $\begin{array}{l}\text { *. } \\
\text { à }\end{array}$ & $\stackrel{*}{\stackrel{*}{c}}$ & 7 & 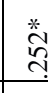 & $\begin{array}{l}\text { *. } \\
\text { के } \\
\text { त्र }\end{array}$ & 范 & 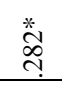 & 誉 & 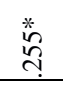 & $\stackrel{*}{\stackrel{*}{ }}$ & 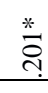 \\
\hline 2 & 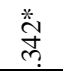 & & 䙦 & $\stackrel{*}{\stackrel{*}{g}}$ & $\begin{array}{l}* \\
\stackrel{\sigma}{\sigma}\end{array}$ & $\begin{array}{l}* \\
\stackrel{*}{5} \\
n\end{array}$ & 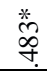 & \begin{tabular}{l}
$*$ \\
\multirow{2}{o}{} \\
$\stackrel{o}{o}$
\end{tabular} & 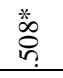 & $\begin{array}{l}* \\
\stackrel{*}{f} \\
\stackrel{f}{f}\end{array}$ & 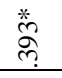 & $\begin{array}{l}\text { *. } \\
\text { ल }\end{array}$ & ले & $\begin{array}{l}\text { \%. } \\
\text { ले }\end{array}$ & \begin{tabular}{l}
$*$ \\
\multirow{\Im}{\Im}{} \\
.
\end{tabular} & $\begin{array}{l}* \\
\stackrel{*}{n} \\
n\end{array}$ & 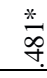 & 囦 & 䔲 & 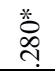 & $\begin{array}{l}* \\
\text { *a } \\
\text { ले }\end{array}$ & $\stackrel{*}{m}$ & ণ্] & 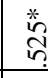 & $\begin{array}{l}* \\
\stackrel{*}{7}\end{array}$ & $\begin{array}{l}* \\
\text { ले } \\
\text { m. }\end{array}$ & $\begin{array}{l}* \\
\stackrel{*}{\circ} \\
\stackrel{0}{0}\end{array}$ & $\begin{array}{l}\stackrel{*}{\sim} \\
\stackrel{0}{f}\end{array}$ & $\begin{array}{l}\text { *. } \\
\text { ฯ }\end{array}$ & $\begin{array}{l}* \\
\text { o } \\
\text { q }\end{array}$ & $\stackrel{*}{m}$ \\
\hline 3 & $\frac{*}{\mathrm{~m}}$ & 热 & & \%) & $\stackrel{*}{\stackrel{*}{g}}$ & 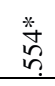 & 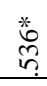 & \begin{tabular}{l}
$*$ \\
\multirow{2}{*}{} \\
f
\end{tabular} & 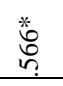 & 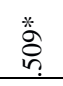 & 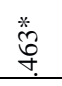 & 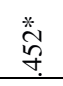 & $\stackrel{*}{\stackrel{*}{7}}$ & 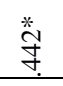 & 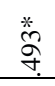 & 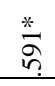 & $\begin{array}{l}* \\
\text { in } \\
\end{array}$ & 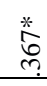 & 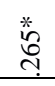 & $\begin{array}{l}\text { * } \\
\text { ते } \\
\text { ? }\end{array}$ & $\begin{array}{l}* \\
\infty \\
q \\
\end{array}$ & 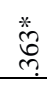 & $\begin{array}{l}\text { *ै } \\
\text { הat } \\
\end{array}$ & 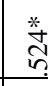 & 苂 & $\begin{array}{l}* \\
\infty \\
\infty \\
\cdots\end{array}$ & $\begin{array}{l}* \\
\stackrel{*}{g} \\
\end{array}$ & $\begin{array}{l}* \\
\text { o } \\
\text { n. }\end{array}$ & $\begin{array}{l}* \\
\text { : } \\
\text { n. }\end{array}$ & $\begin{array}{l}\text { \% } \\
\text { qे }\end{array}$ & के \\
\hline 4 & 卷 & $\frac{*}{\mathcal{g}}$ & 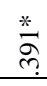 & & $\begin{array}{l}\text { 总 } \\
\text { n? }\end{array}$ & 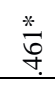 & 剙 & 蒿 & $\begin{array}{l}\text { *. } \\
\text { f. }\end{array}$ & $\begin{array}{l}\stackrel{*}{*} \\
\stackrel{n}{n}\end{array}$ & 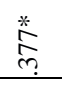 & 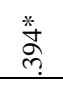 & 莡 & 菐 & $\stackrel{\text { * }}{m}$ & $\begin{array}{l}\text { \%े } \\
\text { ๙ે }\end{array}$ & $\stackrel{*}{\stackrel{*}{g}}$ & $\begin{array}{l}\text { \%े } \\
\stackrel{\text { }}{n}\end{array}$ & 蒙 & $\stackrel{*}{\beth}$ & $\stackrel{*}{m}$ & 苋 & ก & 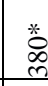 & 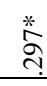 & 蒂 & 苍 & $\stackrel{*}{=}$ & $\stackrel{*}{\stackrel{*}{m}}$ & 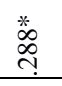 & $\begin{array}{l}* \\
\text { *ै } \\
\text { त्र }\end{array}$ \\
\hline 5 & 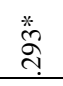 & $\begin{array}{l}\text { *ै. } \\
\stackrel{\sigma}{\sigma}\end{array}$ & $\stackrel{*}{\underset{\gamma}{\gamma}}$ & $\begin{array}{l}\text { 总 } \\
\text { n. }\end{array}$ & & $\begin{array}{l}\text { * } \\
\text { h. } \\
\text { n. }\end{array}$ & 卷 & $\underset{⿱ 乛}{\stackrel{*}{c}}$ & 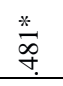 & $\stackrel{*}{\stackrel{*}{7}}$ & $\begin{array}{l}\text { 苂 } \\
\text { ợ }\end{array}$ & $\begin{array}{l}\text { *ैं } \\
\text { 号 }\end{array}$ & 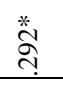 & 荾 & $\stackrel{*}{\stackrel{*}{m}}$ & 䓂 & 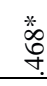 & 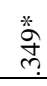 & 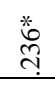 & 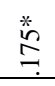 & 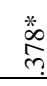 & 苂 & * & 莸 & 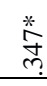 & 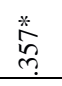 & సે & 苍 & $\begin{array}{l}\text {. } \\
\text { in } \\
\text { ch }\end{array}$ & 苋 & $\begin{array}{l}\text { *ैं } \\
\text { त्र }\end{array}$ \\
\hline 6 & 养 & 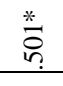 & $\begin{array}{l}\text { 嚄 } \\
\text { in }\end{array}$ & $\begin{array}{l}\frac{*}{6} \\
\\
\end{array}$ & 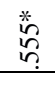 & & $\begin{array}{l}\stackrel{*}{*} \\
\hat{n} \\
\end{array}$ & $\begin{array}{l}* \\
n \\
n\end{array}$ & $\begin{array}{l}\text { *. } \\
\text { 总 }\end{array}$ & $\begin{array}{l}* \\
\text { : } \\
\text { ?n? }\end{array}$ & $\begin{array}{l}\text { *0. } \\
\text { \% }\end{array}$ & $\begin{array}{l}\text { *. } \\
\text { o } \\
\stackrel{0}{f}\end{array}$ & \begin{tabular}{l} 
* \\
品 \\
\multirow{f}{*}{}
\end{tabular} & $\stackrel{*}{\stackrel{*}{7}}$ & 券 & 尊 & $\begin{array}{l}* \\
\text { in } \\
\text { nn }\end{array}$ & $\begin{array}{l}\text { *. } \\
\text { on } \\
\text { f? }\end{array}$ & $\begin{array}{l}\text { *. } \\
\text { त̣ }\end{array}$ & $\begin{array}{l}\text { *. } \\
\text { तె }\end{array}$ & $\begin{array}{l}* \% \\
\text { \% } \\
\end{array}$ & $\stackrel{\text { * }}{\stackrel{*}{7}}$ & $\begin{array}{l}\text { *ैं } \\
\text { â }\end{array}$ & 券 & $\stackrel{*}{\stackrel{*}{?}}$ & 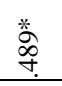 & $\underset{f}{*}$ & 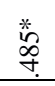 & $\frac{*}{\sigma}$ & $\begin{array}{l}\text { *.ं } \\
\text { f̣ }\end{array}$ & $\frac{*}{\vec{\sigma}}$ \\
\hline 7 & \begin{tabular}{l}
$*$ \\
0 \\
0 \\
\hdashline \\
\end{tabular} & 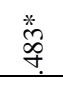 & 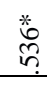 & 卷 & 卷 & $\begin{array}{l}* \\
\stackrel{*}{n} \\
i n\end{array}$ & & $\begin{array}{l}* \\
\text { in } \\
n \\
\end{array}$ & $\begin{array}{l}\frac{*}{*} \\
\substack{\infty \\
?} \\
\end{array}$ & $\begin{array}{l}* \\
.0 \\
0 \\
0\end{array}$ & \begin{tabular}{l}
$*$ \\
\multirow{2}{*}{} \\
$i$
\end{tabular} & $\stackrel{*}{\stackrel{*}{n}}$ & \begin{tabular}{l} 
*. \\
: \\
\hdashline
\end{tabular} & 蒙 & 尊 & 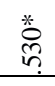 & $\begin{array}{l}* \\
\cdots \\
n\end{array}$ & 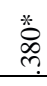 & \begin{tabular}{l}
$*$ \\
$\stackrel{*}{3}$ \\
\cline { 1 - 1 }
\end{tabular} & 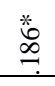 & \begin{tabular}{l}
$*$ \\
$\stackrel{*}{2}$ \\
\multirow{y}{*}{}
\end{tabular} & \begin{tabular}{l}
$*$ \\
\multirow{2}{*}{} \\
$\stackrel{f}{+}$
\end{tabular} & 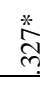 & \begin{tabular}{|l}
$*$ \\
\multirow{2}{*}{} \\
$\stackrel{2}{y}$
\end{tabular} & $\frac{*}{\sigma}$ & $\begin{array}{l}\text { * } \\
\text { ch }\end{array}$ & 犿 & $\stackrel{*}{m}$ & $\begin{array}{l}* \\
\text { 足 } \\
\text { ? }\end{array}$ & $\begin{array}{l}\text { 我 } \\
\stackrel{+}{+}\end{array}$ & $\stackrel{*}{\stackrel{*}{\sigma}}$ \\
\hline 8 & $\stackrel{*}{\frac{*}{c}}$ & $\begin{array}{l}\text { 善 } \\
\text { o }\end{array}$ & 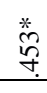 & 䍘 & $\stackrel{\text { H }}{\stackrel{*}{*}}$ & 蒡 & 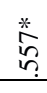 & & 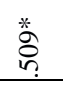 & $\underset{f}{\stackrel{*}{f}}$ & 萦 & 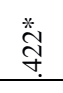 & 尊 & 卷 & 类 & 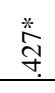 & $\begin{array}{l}\text { : } \\
\text {. } \\
\text { ?. }\end{array}$ & 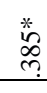 & 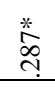 & $\stackrel{*}{\stackrel{*}{ก}}$ & $\begin{array}{l}\text { *.ं. } \\
\stackrel{*}{\circ}\end{array}$ & $\stackrel{*}{\stackrel{*}{c}}$ & 芦 & $\frac{*}{\sigma}$ & 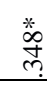 & $\stackrel{*}{\vec{y}}$ & 恖 & 巻 & $\stackrel{*}{\exists}$ & 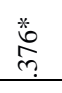 & 恖 \\
\hline 9 & 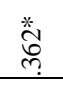 & $\begin{array}{l}* \\
\stackrel{*}{0} \\
\stackrel{n}{n}\end{array}$ & $\begin{array}{l}* \\
: \\
:\end{array}$ & $\begin{array}{l}* \\
\stackrel{*}{\%} \\
\end{array}$ & $\begin{array}{l}\frac{*}{0} \\
\text { of } \\
\end{array}$ & $\begin{array}{l}\text { * } \\
\text { 总 } \\
\end{array}$ & $\begin{array}{l}* \\
\infty \\
i \\
\end{array}$ & $\begin{array}{l}\text { *. } \\
\text { ڤn } \\
\text { in }\end{array}$ & & 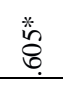 & 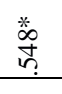 & $\frac{*}{\sigma}$ & $\stackrel{*}{\vec{F}}$ & $\begin{array}{l}* \\
\hat{n} \\
n\end{array}$ & 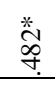 & 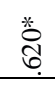 & $\begin{array}{l}* \\
0 \\
\end{array}$ & \begin{tabular}{l}
$*$ \\
\multirow{2}{*}{} \\
\multirow{\gamma}{*}{}
\end{tabular} & $\frac{*}{m}$ & $\begin{array}{l}* \\
\text { \% } \\
0 \\
0\end{array}$ & $\begin{array}{l}* \\
\cdots \\
\widehat{B}\end{array}$ & $\begin{array}{l}\frac{*}{\infty} \\
\stackrel{m}{m}\end{array}$ & 芦 & 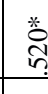 & $\stackrel{*}{\exists}$ & $\begin{array}{l}* \\
\stackrel{*}{*} \\
f\end{array}$ & $\begin{array}{l}* \\
\stackrel{*}{*} \\
n\end{array}$ & $\begin{array}{l}* \\
\stackrel{*}{\sigma} \\
\end{array}$ & $\frac{*}{\sigma}$ & \begin{tabular}{l} 
券 \\
$\infty$ \\
\hdashline
\end{tabular} & $\begin{array}{l}\text { * } \\
6 \\
0\end{array}$ \\
\hline 10 & 思 & $\begin{array}{l}* \\
\stackrel{*}{f} \\
f\end{array}$ & $\begin{array}{l}\text { \%े } \\
\text { مे }\end{array}$ & $\begin{array}{l}\text { *. } \\
\stackrel{c}{m}\end{array}$ & $\begin{array}{l}\text { *. } \\
\stackrel{n}{g} \\
\end{array}$ & $\begin{array}{l}\text { *0. } \\
\text { 号 } \\
\text { n? }\end{array}$ & 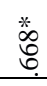 & \begin{tabular}{l}
$*$ \\
\multirow{2}{*}{} \\
\multirow{f}{*}{}
\end{tabular} & $\begin{array}{l}* \\
0 \\
0 \\
0\end{array}$ & & $\begin{array}{l}\text { *. } \\
\text { fot } \\
\end{array}$ & $\begin{array}{l}. \\
\\
\end{array}$ & $\begin{array}{l}\text { 苛 } \\
\text { !n }\end{array}$ & 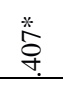 & 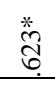 & $\begin{array}{l}* \\
n \\
n \\
n \\
n\end{array}$ & $\stackrel{*}{\pi}$ & $\frac{*}{7}$ & $\begin{array}{l}\text { *. } \\
\text { Oे }\end{array}$ & .: & $\begin{array}{l}* \\
\text { J } \\
\end{array}$ & $\begin{array}{l}\text { 芦 } \\
\stackrel{9}{q}\end{array}$ & 漷 & \begin{tabular}{l}
$*$ \\
\multirow{2}{*}{} \\
\multirow{2}{*}{}
\end{tabular} & $\begin{array}{l}* \\
\stackrel{*}{q} \\
\end{array}$ & $\begin{array}{l}\stackrel{*}{*} \\
\exists \\
\end{array}$ & $\begin{array}{l}\stackrel{*}{*} \\
\stackrel{h}{g} \\
\end{array}$ & ثِ & $\begin{array}{l}* \\
0 \\
0 \\
0\end{array}$ & $\begin{array}{l}\text { \% } \\
\text { bे } \\
\text { y. }\end{array}$ & 芦 \\
\hline 11 & $\stackrel{*}{\frac{*}{m}}$ & \% & 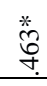 & $\begin{array}{l}\text { 总 } \\
\text { c }\end{array}$ & $\begin{array}{l}* \\
\stackrel{*}{o} \\
q\end{array}$ & 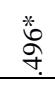 & \begin{tabular}{l}
$*$ \\
\multirow{2}{*}{} \\
$i$
\end{tabular} & $\begin{array}{l}* \\
\text { \% } \\
\stackrel{9}{9}\end{array}$ & 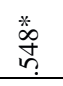 & 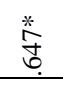 & & \begin{tabular}{l}
$*$ \\
\multirow{2}{*}{} \\
$\hat{n}$
\end{tabular} & $\begin{array}{l}\text { 券 } \\
\text { 字 }\end{array}$ & 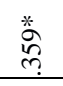 & $\begin{array}{l}\text { \% } \\
\text { वे }\end{array}$ & $\stackrel{*}{m}$ & $\begin{array}{l}* \\
\text { 岁 }\end{array}$ & $\stackrel{*}{\vec{q}}$ & $\begin{array}{l}* \\
\text { \% } \\
\text { â? }\end{array}$ & $\stackrel{*}{\stackrel{*}{ \pm}}$ & \% & $\frac{*}{g}$ & ले & $\begin{array}{l}\frac{*}{7} \\
\end{array}$ & 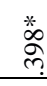 & 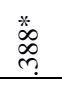 & $\underset{\partial}{\stackrel{*}{\partial}}$ & $\begin{array}{l}* \\
\text { 吾 } \\
\text { in }\end{array}$ & $\begin{array}{l}* \\
\text { \% } \\
\text { n? }\end{array}$ & $\begin{array}{l}\text { \% } \\
\stackrel{*}{y}\end{array}$ & $\begin{array}{l}* \\
\stackrel{*}{o} \\
\end{array}$ \\
\hline 12 & $\frac{* \infty}{m}$ & $\begin{array}{l}\text { *. } \\
\text { ले }\end{array}$ & 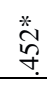 & \% & $\begin{array}{l}\text { *.े } \\
\text { 吕 }\end{array}$ & $\begin{array}{l}* \\
\infty \\
\infty \\
\infty \\
+\end{array}$ & $\begin{array}{l}\text { 券 } \\
\text { !n }\end{array}$ & 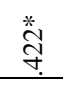 & $\frac{*}{\sigma}$ & $\begin{array}{l}* \\
\\
\end{array}$ & \begin{tabular}{l}
$*$ \\
\multirow{2}{*}{} \\
$i n$ \\
$n$
\end{tabular} & & $\begin{array}{l}\text { 苦 } \\
\text { n. }\end{array}$ & $\begin{array}{l}\text { \% } \\
\text { ळे }\end{array}$ & 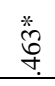 & 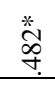 & $\frac{*}{g}$ & 巻 & $\begin{array}{l}\text { *ै } \\
\text { ते }\end{array}$ & $\stackrel{*}{\sigma}$ & $\begin{array}{l}* \\
\stackrel{*}{\sigma} \\
\end{array}$ & $\stackrel{*}{\underset{7}{7}}$ & 卷 & 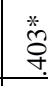 & $\begin{array}{l}\text { *. } \\
\stackrel{*}{\oplus} \\
\end{array}$ & 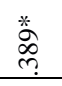 & 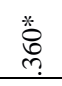 & $\underset{⿱ 乛}{*}$ & $\begin{array}{l}* \\
\stackrel{*}{*} \\
f\end{array}$ & 芦 & 莪 \\
\hline 13 & 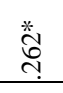 & ले & $\stackrel{*}{\stackrel{*}{\sigma}}$ & 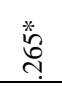 & సิ่ & \begin{tabular}{l}
$*$ \\
\multirow{2}{*}{} \\
\multirow{\sigma}{*}{}
\end{tabular} & 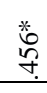 & 券 & $\stackrel{*}{\Rightarrow}$ & 荾 & $\begin{array}{l}\text { 巻 } \\
\text { ô }\end{array}$ & 卷 & & 总 & 荌 & 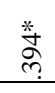 & $\stackrel{*}{\stackrel{*}{c}}$ & 菅 & 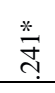 & 草 & 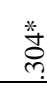 & \begin{tabular}{l} 
*. \\
\multirow{\gamma}{*}{}
\end{tabular} & 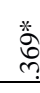 & $\begin{array}{l}* \\
\infty \\
\infty \\
c\end{array}$ & $\begin{array}{l}* \\
\stackrel{*}{*} \\
\stackrel{c}{c}\end{array}$ & 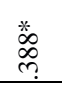 & 芦 & 奠 & 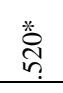 & 囦 & 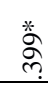 \\
\hline 14 & 卷 & 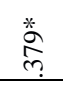 & $\begin{array}{l}* \\
\text { 学 }\end{array}$ & $\begin{array}{l}* \\
\text { \% } \\
\end{array}$ & 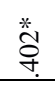 & $\stackrel{*}{\stackrel{*}{7}}$ & \begin{tabular}{l}
$*$ \\
\multirow{8}{+}{} \\
\end{tabular} & 誊 & 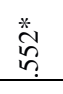 & 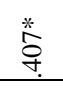 & 卷 & 蒿 & 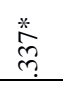 & & \begin{tabular}{l}
$*$ \\
$\stackrel{*}{*}$ \\
\multirow{T}{*}{}
\end{tabular} & \begin{tabular}{l}
$*$ \\
\multirow{2}{*}{} \\
$\mathfrak{n}$
\end{tabular} & \begin{tabular}{|l}
$*$ \\
: \\
\end{tabular} & 券 & $\begin{array}{l}* \\
\text { H. } \\
\text { ते } \\
\end{array}$ & $\stackrel{*}{\stackrel{*}{\sigma}}$ & 룰 & $\begin{array}{l}\text { *ैं } \\
\text { ते } \\
\end{array}$ & ণิ & \begin{tabular}{|c|c}
$*$ \\
$\infty$ \\
\multirow{2}{*}{} \\
\end{tabular} & $\begin{array}{l}\text { *. } \\
\text { ते }\end{array}$ & $\begin{array}{l}* \\
\text { లై } \\
\text { m. }\end{array}$ & 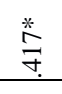 & $\begin{array}{l}\stackrel{*}{h} \\
\end{array}$ & $\stackrel{*}{\stackrel{*}{7}}$ & 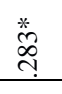 & \begin{tabular}{l}
$*$ \\
\multirow{2}{*}{} \\
\end{tabular} \\
\hline 15 & 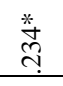 & 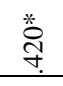 & $\begin{array}{l}\text { 苂 } \\
\stackrel{\sigma}{\sigma}\end{array}$ & $\frac{*}{m}$ & $\stackrel{*}{\stackrel{*}{c}}$ & 券 & 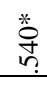 & $\begin{array}{l}* \\
\text { \%o } \\
\text { 子 }\end{array}$ & 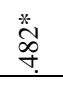 & 荡 & $\begin{array}{l}\text { *. } \\
\text { oे }\end{array}$ & 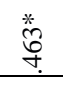 & 鹈 & $\begin{array}{l}\text { *.ँّ } \\
\text {. }\end{array}$ & $i$ & $\begin{array}{l}* \\
\text { N̂ } \\
\text { nn? }\end{array}$ & 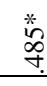 & 卷 & 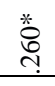 & $\frac{*}{\stackrel{*}{c}}$ & $\frac{*}{\partial}$ & $\stackrel{*}{\stackrel{*}{\vec{n}}}$ & ** & 肎 & $\frac{*}{m}$ & $\stackrel{*}{\stackrel{*}{m}}$ & $\begin{array}{l}* \\
\infty \\
\infty \\
\cdots\end{array}$ & 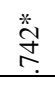 & 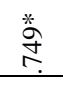 & \begin{tabular}{l}
$*$ \\
\multirow{2}{*}{} \\
$i$ \\
$n$
\end{tabular} & $\frac{*}{n}$ \\
\hline 16 & 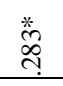 & \begin{tabular}{l}
$*$ \\
\multirow{2}{*}{} \\
$n$
\end{tabular} & $\begin{array}{l}* \\
\stackrel{*}{n}\end{array}$ & 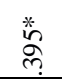 & 蒿 & 害 & $\begin{array}{l}\text { * } \\
\text { 总 } \\
\text { in }\end{array}$ & $\begin{array}{l}* \\
\stackrel{*}{y} \\
y\end{array}$ & 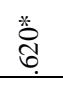 & \begin{tabular}{l}
$*$ \\
\multirow{2}{n}{} \\
$n$
\end{tabular} & $\stackrel{*}{\stackrel{*}{n}}$ & $\begin{array}{l}\stackrel{*}{*} \\
\stackrel{\infty}{+}\end{array}$ & 卷 & 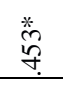 & $\begin{array}{l}* \\
\text { ñ } \\
\text { nै }\end{array}$ & & $\stackrel{*}{5}$ & $\begin{array}{l}* \\
\stackrel{*}{q} \\
q\end{array}$ & $\begin{array}{l}* \\
\text { * } \\
\text { ते }\end{array}$ & 卷 & 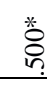 & 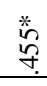 & ${ }^{*}$ & $\begin{array}{l}* \\
6 \\
6\end{array}$ & $\stackrel{*}{\stackrel{*}{f}}$ & $\frac{*}{\vartheta}$ & $\begin{array}{l}* \\
\stackrel{*}{\sigma}\end{array}$ & 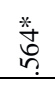 & 卷 & $\stackrel{*}{*}$ & $\begin{array}{l}* \\
\stackrel{*}{*} \\
\stackrel{9}{q}\end{array}$ \\
\hline
\end{tabular}




\begin{tabular}{|c|c|c|c|c|c|c|c|c|c|c|c|c|c|c|c|c|c|c|c|c|c|c|c|c|c|c|c|c|c|c|c|}
\hline 17 & $\stackrel{*}{\stackrel{*}{m}}$ & 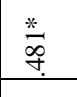 & 雚 & $\begin{array}{l} \\
\\
\end{array}$ & 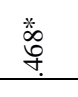 & 奠 & 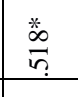 & 总 & 蒿 & $\begin{array}{l}* \\
i \\
i n\end{array}$ & 桨 & \begin{tabular}{|l}
$*$ \\
\end{tabular} & $\stackrel{*}{\stackrel{*}{e}}$ & 葛 & 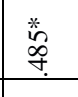 & $\begin{array}{l}* \\
\\
\end{array}$ & & 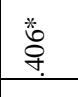 & 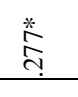 & 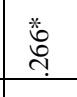 & 峟 & $\begin{array}{l}\text { 类 } \\
\infty \\
\infty\end{array}$ & 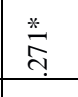 & $\begin{array}{l}* \\
0 \\
0 \\
0\end{array}$ & 范 & \begin{tabular}{l}
$* *$ \\
\multirow{2}{*}{} \\
\multirow{2}{*}{}
\end{tabular} & $\begin{array}{l}\text { 巻 } \\
\text { f. }\end{array}$ & \begin{tabular}{|l}
\multirow{2}{*}{} \\
学 \\
\end{tabular} & 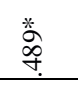 & 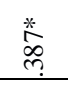 & 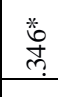 \\
\hline 18 & 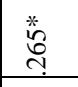 & $\begin{array}{l}\text { 葥 } \\
\end{array}$ & 罳 & 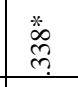 & $\begin{array}{l}\text { 蒽 } \\
\end{array}$ & 曽 & 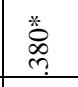 & 恖 & 畹 & \begin{tabular}{l} 
巻 \\
\multirow{\gamma}{*}{}
\end{tabular} & $\frac{*}{\bar{p}}$ & 芦 & 荤 & 兽 & 㟢 & \begin{tabular}{l} 
离 \\
\multirow{q}{*}{}
\end{tabular} & 荌 & & 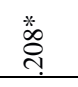 & 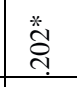 & 茪 & 惫 & 善 & 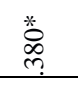 & 善 & $\begin{array}{l}\text { 蔩 } \\
\end{array}$ & 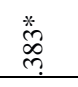 & \begin{tabular}{l} 
并 \\
\multirow{2}{*}{}
\end{tabular} & 莆 & 蒿 & 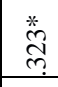 \\
\hline 19 & \begin{tabular}{|l} 
** \\
î̀
\end{tabular} & $\stackrel{*}{\stackrel{*}{9}}$ & 蒙 & 菅 & 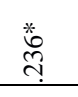 & 商 & 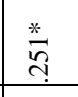 & 网 & $\frac{*}{\mathrm{~m}}$ & 尊 & 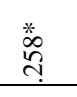 & 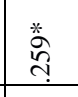 & $\stackrel{*}{\vec{y}}$ & 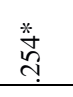 & 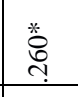 & 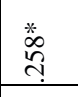 & 卷 & 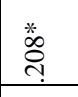 & & 莟 & 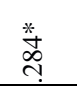 & 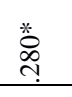 & 華 & 等 & 密 & 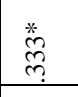 & 苂 & 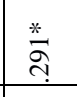 & 芦 & 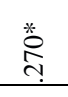 & 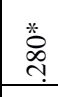 \\
\hline 20 & 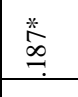 & 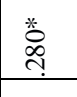 & 䔲 & $\stackrel{*}{\stackrel{*}{\Xi}}$ & 蒽 & 落 & \begin{tabular}{|l}
$*$ \\
$\stackrel{*}{\infty}$ \\
\end{tabular} & $\stackrel{*}{\tilde{n}}$ & 茟 & $\begin{array}{l}\text { *.ंّ } \\
\end{array}$ & $\stackrel{*}{\stackrel{*}{\Xi}}$ & $\frac{*}{9}$ & $\stackrel{*}{*}$ & $\stackrel{*}{\stackrel{*}{\prime}}$ & \begin{tabular}{|l}
$*$ \\
$\stackrel{*}{*}$ \\
\end{tabular} & 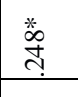 & 军 & 苹 & 晜 & & 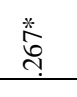 & 䓠 & \begin{tabular}{|l}
$\stackrel{*}{*}$ \\
$\stackrel{*}{\oplus}$ \\
\end{tabular} & 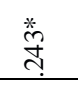 & 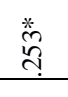 & $\begin{array}{l}\text { \% } \\
\stackrel{*}{d} \\
\end{array}$ & 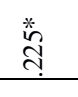 & 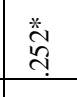 & $\underset{7}{\stackrel{*}{*}}$ & 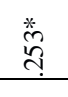 & 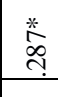 \\
\hline 21 & 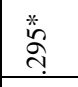 & 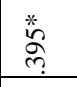 & 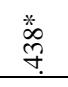 & $\frac{*}{\mathrm{~m}}$ & 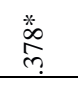 & 善 & 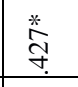 & 恖 & 范 & 永 & 荌 & 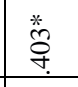 & 铲 & 茪 & 尊 & 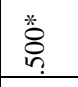 & 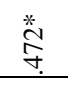 & 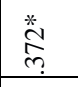 & 惫 & \begin{tabular}{|l} 
菢 \\
\end{tabular} & & 揱 & 惫 & 芯 & 荌 & 蒡 & $\frac{w_{n}^{2}}{n ! n}$ & 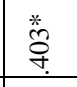 & 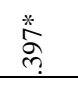 & 䓂 & $\begin{array}{l}* \\
\stackrel{*}{*} \\
\end{array}$ \\
\hline 22 & 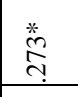 & $\frac{*}{\infty}$ & 商 & 总 & 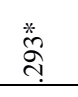 & 㹲 & \begin{tabular}{|l}
\multirow{2}{*}{} \\
它
\end{tabular} & $\stackrel{*}{2}$ & 娄 & $\begin{array}{l}\text { 装 } \\
\text { 子 }\end{array}$ & 卷 & 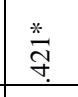 & \begin{tabular}{l} 
苂 \\
\multirow{\gamma}{*}{}
\end{tabular} & 莃 & $\begin{array}{l}\text { 券 } \\
\text { के }\end{array}$ & 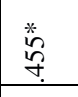 & 类 & 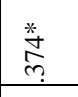 & 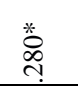 & 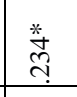 & 淾 & & 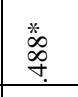 & 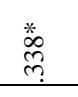 & 芲 & $\begin{array}{l}\text { 卷 } \\
\text { ले }\end{array}$ & 总 & 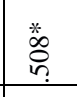 & $\frac{*}{\partial}$ & 卷 & 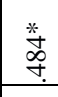 \\
\hline 23 & $\stackrel{*}{\stackrel{*}{\circ}}$ & 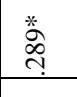 & 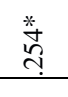 & 券 & స્ํํ & 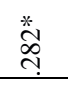 & \begin{tabular}{|l} 
恖 \\
ले
\end{tabular} & 总 & $\stackrel{*}{\mathrm{~m}}$ & 首 & 晜 & 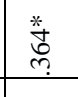 & 总 & 荢 & 恖 & 美 & 卷 & 善 & $\stackrel{*}{\stackrel{*}{\leftrightharpoons}}$ & $\begin{array}{l}\stackrel{*}{*} \\
\stackrel{*}{\sim}\end{array}$ & 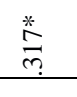 & 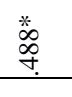 & & $\begin{array}{l}\text { *. } \\
\text { वें }\end{array}$ & लैं & 卷 & 范 & 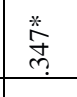 & 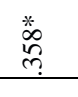 & 䔲 & 善 \\
\hline 24 & 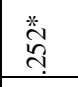 & 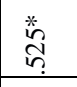 & 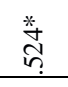 & \begin{tabular}{|l} 
恖 \\
$\stackrel{c}{\circ}$
\end{tabular} & $\begin{array}{l}\text { 䓺 } \\
\text { f. }\end{array}$ & 券 & $\begin{array}{l}\text { 浐 } \\
\text { fy }\end{array}$ & 䓂 & 总 & 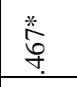 & 苾 & 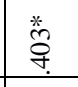 & $\begin{array}{l}* \\
\substack{\infty \\
\infty \\
\infty}\end{array}$ & \begin{tabular}{l} 
象 \\
\multirow{f}{*}{}
\end{tabular} & 糔 & $\begin{array}{l}\text { 善 } \\
\end{array}$ & 商 & 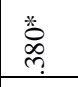 & 前 & $\begin{array}{l}\text { 落 } \\
\text { 号 }\end{array}$ & 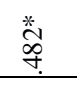 & 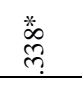 & 养 & & 蒡 & 悉 & $\frac{*}{0}$ & $\begin{array}{l}\frac{*}{2} \\
\stackrel{m}{n}\end{array}$ & 善 & 等。 & $\begin{array}{l}\text { 姜 } \\
\text { م. }\end{array}$ \\
\hline 25 & 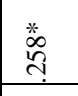 & 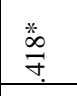 & $\frac{*}{6}$ & 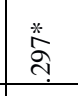 & 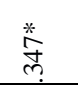 & 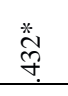 & 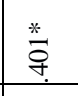 & 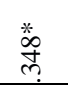 & $\stackrel{*}{F}$ & 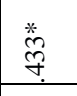 & 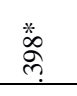 & \begin{tabular}{|l|}
\multirow{2}{*}{} \\
点 \\
\end{tabular} & \begin{tabular}{l} 
巻 \\
\hdashline
\end{tabular} & \begin{tabular}{|l} 
善 \\
\end{tabular} & $\frac{*}{\mathrm{~m}}$ & $\begin{array}{l}* \\
\stackrel{*}{2} \\
\stackrel{f}{+}\end{array}$ & $\stackrel{*}{7} \underset{7}{7}$ & 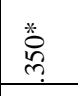 & 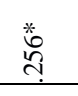 & $\begin{array}{l}\text { * } \\
\hat{i} \\
\end{array}$ & $\begin{array}{l}* \\
\\
F\end{array}$ & $\begin{array}{l}\text { *. } \\
\text { âे } \\
\end{array}$ & 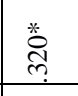 & 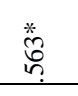 & & 菩 & 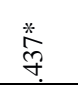 & \begin{tabular}{|l}
\multirow{2}{*}{} \\
ले \\
\end{tabular} & \begin{tabular}{l} 
*. \\
$\infty$ \\
\hdashline
\end{tabular} & $\stackrel{*}{\vec{n}}$ & 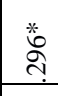 \\
\hline 26 & 卷 & 芦 & 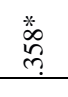 & 噌 & 恖 & 夢 & 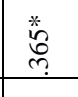 & $\stackrel{*}{\stackrel{*}{q}}$ & 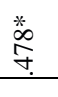 & $\begin{array}{l}* \\
\infty \\
\neq \\
\neq\end{array}$ & 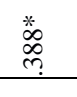 & $\begin{array}{l}\text { \% } \\
\stackrel{*}{\circ} \\
\stackrel{2}{2}\end{array}$ & 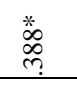 & 䓠 & $\stackrel{*}{\stackrel{*}{m}}$ & 旁 & ส̛. & 管 & 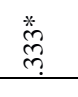 & 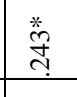 & 萢 & 养 & 卷 & 拳 & 铃 & & 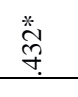 & $\begin{array}{l}* \\
\text { 苂 } \\
\end{array}$ & $\stackrel{*}{F}$ & 善 & 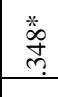 \\
\hline 27 & 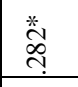 & \begin{tabular}{|l} 
总 \\
品
\end{tabular} & $\stackrel{\frac{*}{m}}{\frac{m}{q}}$ & 总 & 今. & 巻 & 尊 & 卷 & 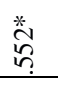 & 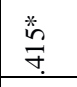 & 巻 & 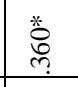 & 总 & $\begin{array}{l}\text { 蒂 } \\
\end{array}$ & 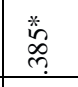 & 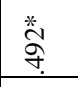 & 善 & 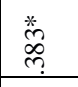 & 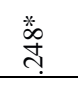 & $\begin{array}{l}\text { 苞 } \\
\text { त्र }\end{array}$ & $\frac{w_{n}^{n}}{n ! n}$ & 善 & 葛 & $\frac{*_{0}^{\circ}}{n ! n}$ & 养 & $\begin{array}{l}\text { 苂 } \\
\text { q. }\end{array}$ & & \begin{tabular}{|l} 
尊 \\
\end{tabular} & 萧 & 善 & \begin{tabular}{|l} 
总 \\
ते
\end{tabular} \\
\hline 28 & 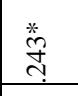 & \begin{tabular}{l}
$*$ \\
Fi \\
\multirow{f}{*}{}
\end{tabular} & 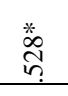 & $\stackrel{*}{=}$ & 总 & 总 & \begin{tabular}{|l|}
$\%$ \\
$m$ \\
$m$ \\
\end{tabular} & 券 & 蓉 & 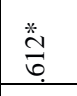 & 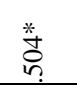 & \begin{tabular}{|l} 
\\
\end{tabular} & 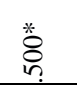 & $\begin{array}{l}\text { 券 } \\
\stackrel{b}{f} \\
\end{array}$ & 茶 & 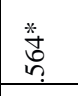 & 芲 & $\begin{array}{l}\frac{*}{\partial} \\
\end{array}$ & 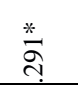 & $\begin{array}{l}\text { *ैं } \\
\text { तે } \\
\end{array}$ & 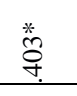 & 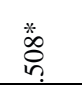 & 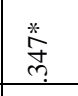 & $\begin{array}{l}* \\
m \\
m \\
m\end{array}$ & 卷 & 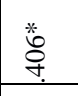 & 尊 & & 莕 & 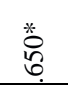 & 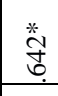 \\
\hline 29 & 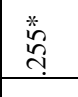 & $\begin{array}{l}\text { *ैं } \\
\text { व̛ } \\
\end{array}$ & 茵 & $\frac{*}{m}$ & 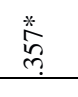 & $\frac{*}{\sigma}$ & 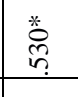 & 美 & $\frac{*}{g}$ & \begin{tabular}{l}
$*$ \\
\multirow{2}{*}{} \\
.
\end{tabular} & 奠 & 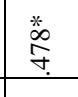 & 萨 & $\begin{array}{l}\stackrel{*}{*} \\
\stackrel{*}{f} \\
\end{array}$ & $\begin{array}{l}\text { 尊 } \\
\end{array}$ & 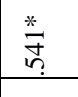 & 养 & 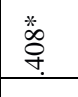 & 卷 & \begin{tabular}{|l} 
*. \\
$\stackrel{2}{c}$ \\
\cline { 1 - 1 }
\end{tabular} & 荡 & 券 & 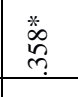 & $\begin{array}{l}\text { 卷 } \\
\text { 点 } \\
\end{array}$ & 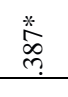 & 学 & $\begin{array}{l}\text { 薄 } \\
\end{array}$ & 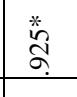 & & 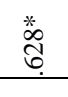 & \begin{tabular}{|l}
$*$ \\
$\cdots$ \\
0 \\
0 \\
\end{tabular} \\
\hline 30 & $\frac{*}{\stackrel{*}{c}}$ & 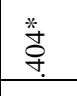 & 因 & 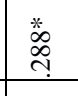 & 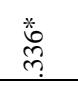 & 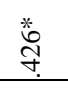 & 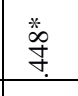 & 总 & 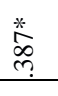 & 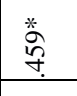 & $\begin{array}{l}\text { 前 } \\
\end{array}$ & 葶 & 菀 & 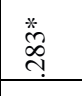 & $\begin{array}{l}\text { 圽 } \\
\vdots \\
\end{array}$ & $\begin{array}{l}\text { 总 } \\
\text { f } \\
\end{array}$ & 养 & \begin{tabular}{|l}
\multirow{\sigma}{*}{$+\frac{1}{9}$} \\
\end{tabular} & 养 & \begin{tabular}{l}
$*$ \\
\multirow{n}{*}{} \\
â \\
\end{tabular} & $\stackrel{*}{\stackrel{*}{c}}$ & 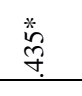 & \begin{tabular}{|l|}
$*$ \\
c. \\
$m$
\end{tabular} & $\begin{array}{l}\text { 巣 } \\
\end{array}$ & $\stackrel{*}{\vec{c}}$ & $\begin{array}{l}\text { \% } \\
\text { 总 } \\
\end{array}$ & 善 & \begin{tabular}{|l}
$*$ \\
0 \\
0 \\
0
\end{tabular} & 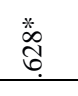 & & \begin{tabular}{l}
$*$ \\
\multirow{6}{*}{} \\
0
\end{tabular} \\
\hline 31 & 蒿 & $\frac{*}{\stackrel{m}{m}}$ & $\frac{*}{\partial}$ & 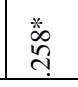 & 谷 & 蒿 & 苍 & 卷 & 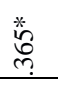 & $\begin{array}{l}\text { 首 } \\
\end{array}$ & 蒾 & 落 & 卷 & 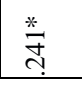 & 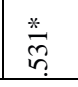 & $\begin{array}{l}\text { 卷 } \\
\text { g }\end{array}$ & 旁 & 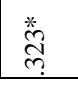 & 莣 & 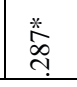 & స్ల్ల & 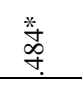 & 总 & 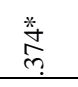 & 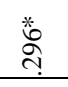 & 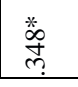 & 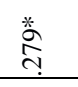 & 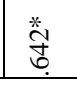 & 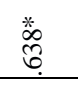 & 带 & \\
\hline
\end{tabular}




\subsection{Construct Validity}

The correlation analysis between PSSQ-29 and the two other self-reported questionnaires was found as expected. The results show that NMRQ is negatively correlated with PSSQ-29 [r=-.500, $\mathrm{p}<.001]$. Similarly, the results of the correlation analysis between PSSQ-29 and ASDS show that the new questionnaire is positively correlated with ASDS [ $\mathrm{r}=$ $.768, \mathrm{p}<.001]$. Figures 1 and 2 present the correlation matrix of the aforementioned results respectively.

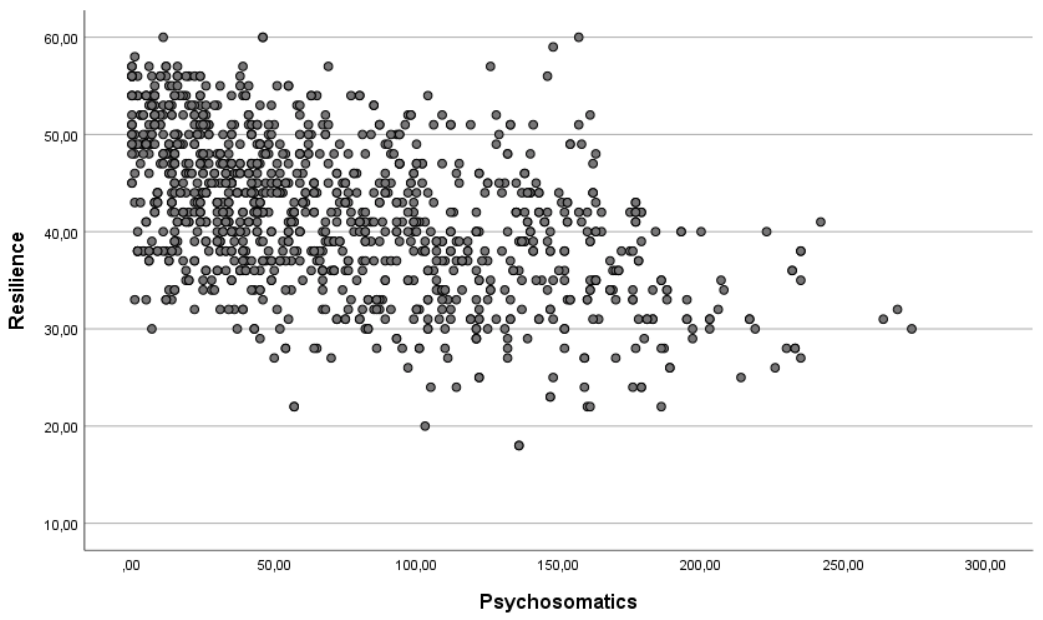

Figure 1. Correlation between Resilience (NMRQ) and Psychosomatics (PSSQ-29).

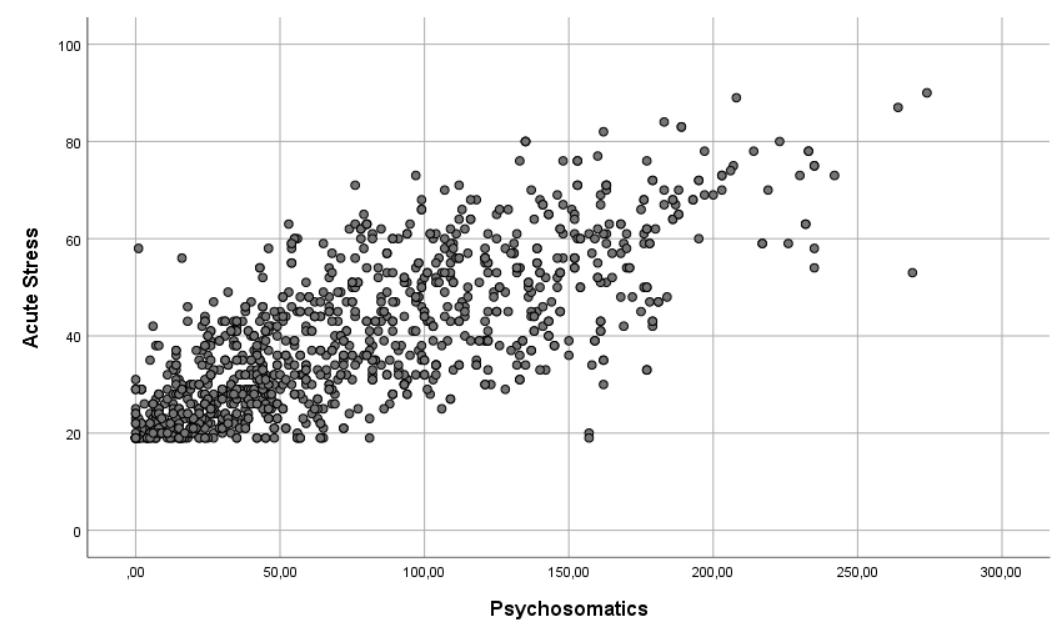

Figure 2. Correlation between ASDS and PSSQ-29.

\subsection{Predictive Validity}

Predictive validity for PSSQ-29 was performed via the use of a linear regression analysis. The analysis showed that the level of ASD, as measured by ASDS, predicted $59 \%$ of the variance of PSSQ-29. It is discussed that a high ratio of prediction in behavioral science starts from about $26 \%$, while the closer this ratio gets to $100 \%$ it may suggest that the two variables measure the same component. ${ }^{[39]}$ It is delineated that the ratio of $59 \%$ is large in effect, as well as that ASDS and PSSQ-29 do not measure the same component. Rather, both scales are based on the biology of the sympathetic nervous adaptation.

\section{DISCUSSION}

\subsection{General Discussion}

In this article the authors showed the analysis that was performed with a view to test the reliability and validity of PSSQ-29. The analysis suggests that PSSQ-29 is both valid and reliable in the Greek population. What is more, it is suggested that PSSQ-29 may be used in clinical practice and research, and thus two main issues need to 
be discussed thoroughly in this part of the article.

Firstly, PSSQ-29 is probably the first tool that contains both mental and somatic symptoms in a single self-reported scale. The field of psychosomatics is probably one of the oldest in the broad field of behavioral sciences, ${ }^{[40]}$ as well as it is the presuccessor of Behavioral Medicine. ${ }^{[41]}$ Thus, a reliable and valid single questionnaire that measures both mental and somatic conditions is considered by the authors of this study as a great necessity in the field of Behavioral Medicine. This is due to the fact that a great deal of current research concerns the role of stress in mental and physical health. Consequently, PSSQ-29 may be used to serve such research designs and provide evidence to such research questions. To support this view, the items of PSSQ-29 were designed based on the idea that stress is the cornerstone of mental, cognitive, affect and somatic symptoms. The mindset behind the development of PSSQ-29 relies to the idea that mental and somatic disorders are one entity, such in the ideology of 'neutral monism' that was initially reflected for the design of PSSQ29.

Secondly, the symptoms and the design of the items of PSSQ-29 are based on two major sources of information that professionals in the broad field of Behavioral Medicine use commonly in clinical practice and research. These concern the Encyclopedia of Behavioral Medicine ${ }^{[11]}$ and ICD-10. ${ }^{[29]}$ It is likely that questionnaires that are designed based on empirical evidence upon the stress response and the sympathetic adaptation measure mostly the symptomatology of psychiatric disorders such as the ASD and 'PostTraumatic Stress Disorder' (PTSD). Those questionnaires may serve better in the hands of mental professionals who are predominantly interested in psychopathy. Additionally, those questionnaires may also neglect significantly the somatic consequences of negative mood and affect, as well as the issue of 'multi-morbidity'.
Indeed, in recent years the term 'multi-morbidity' has been strongly introduced in Behavioral Medicine. ${ }^{[42]}$ In a nutshell, a condition that may reflect accurately the later term is PTSD. A serious amount of studies show that PTSD is a multi-morbid condition, since it concerns for instance equally 'weight gain' issues, ${ }^{[43]}$ 'motor' and 'mood' problems, [44] 'CVD' [45] and 'aggressiveness' [46] amongst many other. PSSQ-29 may illustrate to a professional that beyond weight gain issues, the client deals with negative affect and CVD-related symptoms. To support this idea, a recent meta-analysis ${ }^{[47]}$ clustered 7 main mental and somatic conditions/ disorders that are positively correlated with PTSD in addition to some negatively correlated psychosocial traits. As a consequence, self-reported questionnaires that measure exclusively symptoms of PTSD may neglect that the stress-related condition is likely to be a part of a cluster of disorders, and not a condition that stands on its own. On the contrary, the design of PSSQ-29 is likely to depict more of these multi-morbid mental and somatic symptoms. This use of PSSQ-29 may also be applicable to many conditions that are clustered with a series of somatic disorders including ASD, anxiety and depressive disorders, psychosis and neurodegenerative disorders.

\subsection{Proposed Use of PSSQ-29 \& Future Studies}

First of all, since PSSQ-29 was designed to measure the level of psychosomatic symptoms during the spread of the COVID-19 pandemic in the Greek society, it is of common sense that the new questionnaire can be used as a screening tool of psychosomatic symptoms during a potential new wave of the COVID-19 pandemic in Greece, and probably during any other related pandemic. This provides also some support in using PSSQ-29 into highly stressful situations such as natural disasters, war conflicts, massive immigration and environmental pollutions. 
Considering again the design of PSSQ-29, it is proposed that the new questionnaire may be used by health professional in clinical settings or private practice, as well as in integrated care for the assessment of psychosomatic symptoms. A supportive paradigm was provided in the case of PTSD. For the same reason, PSSQ29 can also compliment other self-reported screening questionnaires in clinical practice. For instance, people who experience mental conditions such as ASD, PTSD, anxiety, depression and alexithymia may also present high levels of psychosomatic symptoms.

Further, the predictive validity analysis of PSSQ-29 shows that stress predicts $59 \%$ of variance in this study. This may suggest that the new psychometric tool measure and depict how stress is expressed in the body. To elaborate, the 29 items of PSSQ-29 include conditions whose rationale of development and classification was based on the activation of the HPA Axis against any stressor/s. For instance, in a N-of-1 study ${ }^{[48,49]}$ someone may respond to stress by eating less, i.e., item 1, and someone else into experiencing more sexual disorders, i.e., item 26. Therefore, stress may result in different mental and somatic symptoms from one person to another.

Additionally, researchers in future studies may translate and adapt the questionnaire in other populations and cultures, as well as in other environmental conditions and circumstances. The design of PSSQ-29 is based on the behavioral expression of biological responses of the HPA Axis, and as a result it is hypothesized that cultural differences will be much limited in comparison to other self-reported psychosocial scales.

In addition, all original items in PSSQ-29 start with the statement 'During the measures against coronavirus, [...]'. Considering that the average days that had passed since the announcement of the mandatory measures against the spread of COVID-19 in the country was found at 33.3 , it is proposed for future studies to replace the original statement of the questions with a one that asks if the participant experience the symptoms throughout a range of 14 to 30 days.

\subsection{Limitations}

With regard to the limitations in the design of the present study, it is worth mentioning that PSSQ-29 was tested exclusively in the Greek population during the COVID-19 outbreak. This practically limited the analysis. A cut-line score is not provided, since the researchers considered that the observed mean scores were likely to be higher due to the COVID-19 pandemic. It is assumed that in everyday life conditions the according mean scores of PSSQ-29 will be lower than the ones in this study.

\section{CONCLUSION}

To recapitulate, the present study presented the analysis that was performed in favor of adapting a newly designed selfreported questionnaire, named PSSQ-29. The background review revealed that most of the previous reliable and valid questionnaires in the field of psychosomatics and Behavioral Medicine do not measure mental and somatic symptoms simultaneously. Hence, the new scale was designed to serve as a selfreported tool that would measure psychosomatic symptoms -mental and somatic- of participants in an epidemiological study that took place in Greece during government's obligatory social and economic measures against the spread of the disease in the state between March 23rd and May 4th of 2020. The results of the current introductory study show high levels of reliability and validity for PSSQ-29. Last but not least, the statistical analysis provides a strong initial support for the use of PSSQ-29 in various settings and circumstances, as well as in academic research in the foreseeable future. 


\section{ACKNOWLEGMENTS}

All authors acknowledge that PSSQ29 was conceived and designed by the lead author of this article, Mr. Georgios Pilafas. The authors also acknowledge the significant contribution of peers, family members, friends and members of the community in this study. Lastly, it is acknowledged the contribution of Mrs. Anastasia Dermatis and Mr. Dimitrios Papaioannou in the data collection process and transfer for statistical analysis.

\section{Conflict of Interest: None}

\section{Source of Funding: None}

Ethical Approval: The study was approved by the Ethics Committee of Research and Conduct of the City Unity College and the City Unity Research Center in Athens, Greece. The approval reference number is 2020PSYRSC-003.

\section{REFERENCES}

1. Ando T. Psychosomatic Disorder. In: Gellman MD, Turner JR, editors. Encyclopedia of Behavioral Medicine. New York, NY: Springer New York; 2013. p. 1588-90.

2. Deter HC, Orth-Gomér K, Wasilewski B, Verissimo R. The European Network on Psychosomatic Medicine (ENPM) - history and future directions. BioPsychoSocial Medicine 2017; 11(1):3.

3. Fava GA, Cosci F, Sonino N. Current Psychosomatic Practice. Psychotherapy and Psychosomatics 2017; 86(1):13-30.

4. Nakao M. Key aims of the special series "the meaning of behavioral medicine in the psychosomatic field." BioPsycho Social Medicine 2016; 10(1):3.

5. Engel G. The need for a new medical model: a challenge for biomedicine. Science 1977; 196(4286):129.

6. Engel GL. The biopsychosocial model and the education of health professionals. General Hospital Psychiatry 1979; $1(2): 156-65$.

7. Wagner NF, Apostolova I. Two Sides of the Same Coin? Neutral Monism as an Attempt to Reconcile Subjectivity and Objectivity in
Personal Identity. Metaphysica 2020; 21(1):129-49.

8. Jin Y. On the Essence of Russell's Neutral Monism. In: Tao, Nature and Man. Springer; 2020. p. 129-37.

9. Silberstein M, Stuckey W. Re-Thinking the World with Neutral Monism: Removing the Boundaries Between Mind, Matter, and Spacetime. Entropy 2020; 22(5).

10. Selye H. The stress of life. In: McGraw-Hill 1956. p. xvi, 324.

11. Gellman M., Turner J. R. (Eds.). Encyclopedia of Behavioral Medicine. Springer-Verlag; 2013.

12. Ernsberger U, Rohrer H. Sympathetic tales: subdivisons of the autonomic nervous system and the impact of developmental studies. Neural Development. 2018; 13(1):20.

13. Cannon WB. Bodily Changes in Pain, Hunger, Fear and Rage: An Account of Recent Researches into the Function of Emotional Excitement. Journal of the American Medical Association 1929; 93(12). p.944-944.

14. Lovallo WR, Buchanan TW. Stress hormones in psychophysiological research: Emotional, behavioral, and cognitive implications. 2017.

15. Folkman S. Stress: Appraisal and Coping. In: Gellman MD, Turner JR, editors. Encyclopedia of Behavioral Medicine. New York, NY: Springer New York; 2013. p. 1913-5.

16. Folkman S, Lazarus RS. Coping as a mediator of emotion. Journal of personality and social psychology 1988; 54(3):466.

17. Lazarus RS. Stress and emotion: A new synthesis. Springer Publishing Co; 1999. p. xiv, 342.

18. Lazarus RS, Folkman S. Transactional theory and research on emotions and coping. Eur J Pers 1987; 1(3):141-69.

19. Fink G. Selye's general adaptation syndrome: stress-induced gastro-duodenal ulceration and inflammatory bowel disease. Journal of Endocrinology 2017; 232(3):F15.

20. Wong A, Zhang B, Jiang M, Gong E, Zhang $\mathrm{Y}$, Lee S. Oxidative stress in acne vulgaris. J Clin Dermatol Ther 2016; 3:020.

21. Kivimäki M, Steptoe A. Effects of stress on the development and progression of cardiovascular disease. Nature Reviews Cardiology 2018; 15(4):215-29. 
22. Kang M, Bang M, Lee SY, Lee E, Yoo SW, An SK. Coping styles in individuals at ultrahigh risk for psychosis: Associations with cognitive appraisals. Psychiatry Research 2018; 264:162-8.

23. Strongylaki N P, Dermati A, Pilafas G, Menti D, Lyrakos G. Effect of Coping Strategies on Acute Stress during the COVID-19 Pandemic in Greece. Health \& Research Journal; in press

24. Goodman LA. Snowball sampling. The annals of mathematical statistics. 1961. p. $148-70$.

25. Palinkas LA, Horwitz SM, Green CA, Wisdom JP, Duan N, Hoagwood K. Purposeful Sampling for Qualitative Data Collection and Analysis in Mixed Method Implementation Research. Administration and Policy in Mental Health and Mental Health Services Research 2015; 42(5): 53344.

26. Comrey AL, Lee HB. A first course in factor analysis. Psychology press; 2013.

27. Anthoine E, Moret L, Regnault A, Sébille V, Hardouin J-B. Sample size used to validate a scale: a review of publications on newly-developed patient reported outcomes measures. Health and Quality of Life Outcomes 2014; 12(1):2.

28. Faul F, Erdfelder E, Buchner A, Lang A-G. Statistical power analyses using $G^{*}$ Power 3.1: Tests for correlation and regression analyses. Behavior Research Methods 2009; 41(4):1149-60.

29. World Health Organization. ICD-10 (Version:2019). 2019

30. Likert R. A technique for the measurement of attitudes. Archives of psychology 1932.

31. Pilafas G, Strongylaki NP, Papaioannou D, Menti D, Lyrakos G. Adaptation of" Nicholson McBride Resilience Questionnaire"(NMRQ) in Greek. A reliability and validity study in an epidemiological Greek sample. Health \& Research Journal 2020; 6(4):123-31.

32. Pilafas G, Strongylaki NP, Menti D, Lyrakos G. Introducing the Greek adaptation of acute stress disorder scale'(ASDS). High reliabil-ity and validity in an epidemiological sample. Health \& Research Journal 2021; 7(2):65-73.

33. IBM Corp. IBM SPSS Statistics for Windows. Armonk, NY: IBM Corp; 2020.

34. Mokkink LB, Terwee CB, Patrick DL, Alonso J, Stratford PW, Knol DL, et al. The
COSMIN study reached international consensus on taxonomy, terminology, and definitions of measurement properties for health-related patient-reported outcomes. Journal of clinical epidemiology 2010; 63(7):737-45.

35. Jendryczko D, Scharfen J, Holling H. The Impact of Situational Test Anxiety on Retest Effects in Cognitive Ability Testing: A Structural Equation Modeling Approach. Journal of Intelligence 2019; 7(4).

36. Lyon AR, Connors E, Jensen-Doss A, Landes SJ, Lewis CC, McLeod BD, et al. Intentional research design in implementation science: implications for the use of nomothetic and idiographic assessment. Translational Behavioral Medicine 2017 ; 7(3):567-80.

37. Gesser-Edelsburg A, Abed Elhadi Shahbari N, Cohen R, Mir Halavi A, Hijazi R, PazYaakobovitch G, et al. Differences in Perceptions of Health Information Between the Public and Health Care Professionals: Nonprobability Sampling Questionnaire Survey. J Med Internet Res 2019; 21(7):e14105.

38. Field A. Discovering statistics using IBM SPSS statistics. Sage; 2013.

39. Miles J. R Squared, Adjusted R Squared. In: Wiley StatsRef: Statistics Reference Online. American Cancer Society 2014.

40. Nisar H, Srivastava R. Fundamental concept of psychosomatic disorders: a review. International Journal of Contemporary Medicine Surgery and Radiology 2018; 3(1):12-8.

41. Engel BT. Psychosomatic medicine, behavioral medicine, just plain medicine. Psychosomatic Medicine 1986; 48(7).

42. Birk JL, Kronish IM, Moise N, Falzon L, Yoon S, Davidson KW. Depression and multimorbidity: Considering temporal characteristics of the associations between depression and multiple chronic diseases. Health Psychology 2019; 38(9):802.

43. Dorflinger LM, Masheb RM. PTSD is associated with emotional eating among veterans seeking treatment for overweight/obesity. Eating Behaviors 2018; 31:8-11.

44. Hoover M. The Benefits of Therapeutic Recreation for Veterans Suffering PTSD. 2017.

45. Beristianos MH, Yaffe K, Cohen B, Byers AL. PTSD and Risk of Incident 
Georgios Pilafas et.al. Introducing the 'psychosomatic screening questionnaire - 29' (PSSQ-29): reliability and validity in an epidemiological sample of 1,158 participants in Greece during the COVID-19 domestic lockdown.

Cardiovascular Disease in Aging Veterans. The American Journal of Geriatric Psychiatry 2016; 24(3):192-200.

46. Taft CT, Creech SK, Murphy CM. Anger and aggression in PTSD. Current Opinion in Psychology 2017; 14:67-71.

47. Nievergelt CM, Maihofer AX, Klengel T, Atkinson EG, Chen C-Y, Choi KW, et al. International meta-analysis of PTSD genome-wide association studies identifies sex- and ancestry-specific genetic risk loci. Nature Communications 2019; 10(1):4558.

48. Fisher AJ. Toward a dynamic model of psychological assessment: Implications for personalized care. Journal of consulting and clinical psychology 2015; 83(4):825.
49. McDonald S, Vieira R, Johnston DW. Analysing N-of-1 observational data in health psychology and behavioural medicine: a 10-step SPSS tutorial for beginners. null 2020; 8(1):32-54.

How to cite this article: Pilafas G, Prouzou A, Strongylaki NP et.al. Introducing the 'psychosomatic screening questionnaire - 29' (PSSQ-29): reliability and validity in an epidemiological sample of 1,158 participants in Greece during the COVID-19 domestic lockdown. Int J Health Sci Res. 2021; 11(6): 5265. DOI: https://doi.org/10.52403/ijhsr. 20210609 